\title{
„Mehr Geschichte wagen“! LUSIR und die ganze Geschichte der Arbeiter im Ruhrgebiet vor, während und nach dem Nationalsozialismus
}

\author{
Franka Maubach
}

Friedrich-Schiller-Universität Jena, Historisches Institut,

Fürstengraben 13, 07743 Jena

franka.maubach@uni-jena.de

\section{Einleitung}

Anfang der achtziger Jahre schwärmte eine Gruppe junger Geschichtsforscher aus, um Arbeiter und Arbeiterinnen sowie Angestellte im Ruhrgebiet über ihre Erfahrungen vor, während und nach dem Nationalsozialismus zu interviewen. ${ }^{1}$ $\mathrm{Zu}$ diesem Zeitpunkt war das Revier längst zu einer uneinnehmbar scheinenden Hochburg der Sozialdemokratie geworden, stabil, ja konservativ links. Das von dem Essener Historiker Lutz Niethammer geleitete Oral-History-Projekt stand unter einem schlicht thematischen, auf den ersten Blick nicht besonders aufregenden Titel: Lebensgeschichte und Sozialkultur im Ruhrgebiet 1930-196o. Einzig der Untersuchungszeitraum, der die üblichen politische Großzäsuren konsequent überschritt und vom Ende der Weimarer Republik bis in die Nachkriegszeit reichte, ließ manchen vielleicht aufhorchen. Projektintern sprachen die Mitarbeiter bald von LUSIR, und unter diesem Namen machte das Interviewprojekt in der zeitgeschichtlichen Zunft die Runde und gelangte zu einiger Berühmtheit. ${ }^{2}$

LUSIR: Das war das erste groß angelegte Oral-History-Projekt in der Bundesrepublik, das stellvertretend stand für den Boom alltags- und lokalgeschichtlicher Zugänge zur Geschichte nicht nur, aber vor allem des Nationalsozialismus. Lokale Akteure in den überall aus dem Boden schießenden Geschichtswerkstätten und -initiativen arbeiteten mit Geschichtslehrern und Historikern

\footnotetext{
1 Es handelt sich um die erweiterte Fassung eines bereits erschienenen Beitrags; vgl. Maubach (2017). Ich danke Anke te Heesen und Dorothea Walzer für ihre Kritik und Unterstützung sowie Ulrich Herbert für sein Feedback und die Zustimmung zum Abdruck der Interviewzitate.

2 Vgl. Niethammer (1986a), S. 25, FN 1: Das Projekt sei „unter uns als LUSIR abgekürzt“ worden.
} 
an den Universitäten zusammen. ${ }^{3}$ Auch LUSIR wuchs in einem solchen lebendigen Milieu kritisch-kollektiver Geschichtsarbeit im Ruhrgebiet, und aus der Sicht seiner Mitarbeiter und Mitarbeiterinnen kam den Interviewpartnern, die sich vor ihre Mikrofone setzten, eine tragende, ja eine ganz aktive Rolle zu, die mit unserem heutigen Bild vom telegen-artifiziellen Zeitzeugen nichts zu tun hat. ${ }^{4}$ Sie fungierten nicht einfach als passive Gedächtnisspeicher, denen für kurze Filmsequenzen oder Radioeinspieler nach Bedarf passende Fragmente, dramatische Anekdoten oder illustrative Details entnommen werden konnten, sondern sollten zu gleichberechtigten Akteuren an der Seite der Wissenschaftler avancieren, als selbstbewusste Subjekte souverän über ihre Geschichte berichten und zudem einen - ihren - Beitrag zum öffentlichen Geschichtsbild leisten. Nicht um kleingeschnittene Gedächtnisstreifen ging es, sondern um die Lebensgeschichten in ihrer Ganzheit. LUSIR war ein programmatisches und zu Beginn auch ein idealistisches Projekt, stellte es doch den Versuch dar, eine aus Sicht der beteiligten Forscher zunehmend konservativ stagnierende Gesellschaft zu redemokratisieren, indem die Menschen nicht nur stärker an der Politik beteiligt wurden, sondern auch an der Geschichte ihrer Zeit. „Kooperative Quellenproduktion“ und „solidarische Geschichtsschreibung" nannte Lutz Niethammer das in seiner Einleitung zum ersten Band und sprach nicht ohne Augenzwinkern von „Beihilfe zur Quellenentstehung“. ${ }^{5}$ Der Anspruch, den Menschen ihre Geschichte zur eigenen Nachlassverwaltung zurückzugeben, war in der linken Geschichtsbewegung verbreitet; in einem Radiointerview im Umfeld des Berliner Historikertags 1984, auf dem heftig über die Relevanz der Alltagsgeschichte gestritten wurde, forderte Detlev Peukert die „Aneignung der eigenen Geschichte durch die Betroffenen selbst“ . ${ }^{6}$ Und in einem im Umfeld von LUSIR entstandenen, von der SPD-Landesregierung unterstützten und 1988 erschienenen Bildtextband zur Ruhrgebietsgeschichte hieß es programmatisch: „Mehr Geschichte wagen“.7 Aber was bedeutete das genau?

Für diese Form einer „solidarischen Geschichtsschreibung“ wählten die durchweg (mehr oder weniger) links eingestellten Historiker mit den Arbeitern und Angestellten im Revier eine Personengruppe aus, mit der sie

3 Vgl. zeitgenössisch Schöttler (1984); jünger Büttner (2009).

4 Vgl. Sabrow/Frei (2012).

5 Niethammer (1986a), S. 17 f. Vgl. auch den Zwischenbericht, in dem von einer „kommunikative[n] Heuristik“ die Rede ist: Archiv „Deutsches Gedächtnis“ an der FernUniversität Hagen, Bestand LUSIR, Ordner „Papers LUSIR Bd. 1 und Zwischenbericht“, Zwischenbericht v. 9.12.1981, 59 Seiten, S. 14.

6 Dokumentiert in Hochmuth (2015).

7 Vgl. Niethammer (1988), Kapiteltitel S. 234. 
sympathisierten. Was die Vertreter der Arbeiterklasse und Angestelltenschicht, ehemalige Kruppianer, Bergleute und Metaller, Sekretärinnen und Köchinnen, den Oral Historians erzählten, waren indes unerhörte Begebenheiten am laufenden Band - bester Novellenstoff: Da gab es einen Arbeiter in der Sozialistischen Arbeiterjugend, der auch schon mal beim Stahlhelm vorbeischaute. Aus der Zeit der großen Auseinandersetzungen am Ende der Weimarer Republik erzählte er als kleine Anekdote, wie er einmal einen befreundeten Stahlhelmer mit seiner Jacke als Tarnung „aus einer von Antifaschisten umzingelten Veranstaltung“ gerettet habe. („Ich bin mit allen gut ausgekommen“, hieß das passende Interviewzitat, mit dem Alexander von Plato seinen Aufsatz zur Auflösung der Arbeitermilieus überschrieb. ${ }^{8}$ ) Eine Köchin empfand den Faschismus gar nicht als Höhepunkt einer entmächtigenden Herrschaft des Patriarchats, sondern als Zeit einer wundersamen Privilegierung gegenüber den wirklich unterdrückten Gruppen wie den Zwangsarbeitern, für die sie in einem Lager zwar kochte, die sie aber als Masse ohne Gesicht und Geschichte beschrieb und gegen die sie bei der Essensausgabe gelegentlich handgreiflich wurde, um die, wie sie sagte „Brotneidischen“ (wahrscheinlich einfach nur Hungrigen) vom Nachschlag abzuhalten - Ulrich Herbert nannte das „Apartheid nebenan “. ${ }^{9}$ Der Zinkzieher Fritz Harenberg erinnerte sich in einer atemlosen, unreflektierten Erzählung an den "Blitzkrieg“ vor allem als Beutezug in Länder des Überflusses, wo ein Schnäppchen auf das andere folgte. ${ }^{10}$ Es gab viele solche Geschichten; sie erstaunten nicht nur, sondern forderten zur Interpretation heraus. Aber wie konnte man sie interpretieren?

Die Geschichte, die aus diesen irritierenden Geschichten destilliert wurde, unterschied sich gänzlich von dem, was man zeitgenössisch unter akademischer Historiografie verstand - und das, obwohl es ausgebildete Historiker und Historikerinnen waren, die sich nun daran machten, die Geschichte der Ruhrarbeiterschaft zu schreiben. Nicht nur rückten neue Themen ins Blickfeld - die Arbeiter-, die Frauengeschichte und allgemein die Geschichte derjenigen, die bis dahin "keine Stimme gehabt" hatten -, es änderten sich auch die Formen der sprachlichen Darstellung. Besonders sinnfällig macht das die Entscheidung, O-Töne aus den Interviews als Titel auf die Cover der drei LUSIR-Bände zu setzen, keine glatten und eingängigen Thesen, sondern sperrige und widersprüchliche Aussagen. So heißt der erste Band Die Jahre weiß man nicht, wo man die heute hinsetzen soll, und der Titel seines Nachfolgers treibt das Verwirrspiel noch weiter: Hinterher weiß man, daß es richtig war, daß es schiefgegangen ist. Schon die atemberaubenden temporalen Verwicklungen verweisen

\footnotetext{
8 von Plato (1986), S. 45 .

9 Herbert (1986), S. 246.

10 Vgl. Niethammer (1986b), S. $170 \mathrm{f}$.
} 
darauf, dass in der mündlichen (Erinnerungs-)Rede verschiedene Zeitebenen unauflöslich ineinander geschoben und nicht so säuberlich voneinander getrennt sind wie in dem Zeitlauf chronologisch folgenden Memoiren. Nur für den dritten Band wählten die Herausgeber, weil die Titel "manchen Lesern zu lang erschienen" waren, einen vergleichsweise kurzen, aber ebenfalls mit der Zeitwahrnehmung der Interviewten spielenden Titel: Wir kriegen jetzt andere Zeiten.11

Wie veränderten sich die Geschichtsvorstellung und -darstellung, wenn man subjektive Erinnerungen in sie einspeiste? Vermochten die erzählten Lebensgeschichten etablierte historiografische Darstellungsweisen herauszufordern? Was geschah mit der großen Geschichte, wenn man sich dazu entschied, sie sich von den einfachen Menschen auf der Straße erzählen zu lassen? Wenn man sich dafür entschied, den Geschehnissen und Anekdoten des Alltags Raum zu geben und ihnen durch Nachfragen bis in den letzten Winkel zu folgen? Was, wenn die Quellen dem Historiker, der sie auswerten wollte, widersprechen konnten? Wenn sie nicht sagten, was dieser erwartete? Bahnte sich die lebendige Sprache mündlicher Erinnerung einen Weg in den distanzierten Stil historiografischer Untersuchungen? Aber auch: Ließen sich die oft widersprüchlichen Erinnerungen der Interviewpartner überhaupt in eine größere Geschichtserzählung integrieren? Waren Alltags- und Erfahrungsgeschichte synthesefähig? Bevor ich diese Fragen zu beantworten versuche, werde ich einleitend den Ansatz der Sekundärauswertung von Interviewquellen vorstellen und LUSIR - nur sehr grob - in seinem historischen Kontext platzieren. Danach untersuche ich allgemein das Erkenntnispotenzial der Lebensgeschichten sowie einzelne, assoziativ erzählte Geschichten. Zum Schluss werde ich - aus einem aktuellen Frageinteresse heraus - Überlegungen dazu anstellen, ob und wie sich aus Erinnerungserzählungen synthetische Darstellungen gewinnen lassen.

\section{Sekundäranalyse als Wissens- und Wissenschaftsgeschichte: nach den Fragen fragen}

Die folgenden Überlegungen zu LUSIR gehören in den Bereich der Sekundäranalyse von Interviews, der in den letzten Jahren zunehmend in den Forschungsfokus rückt. Dabei ist die empirische Sozialforschung anderen Disziplinen und auch der Geschichtswissenschaft voraus, vielleicht, weil sie

11 Vgl. die drei Bände: Niethammer (1986, zuerst 1983; 1983); Niethammer/von Plato (1985). Das Zitat vgl. Niethammer/von Plato (1985a), S. 14. 
schon seit den siebziger Jahren einen ausgefeilten Methodenapparat für die Interviewforschung entwickelt hat. Sozialwissenschaftler und Sozialwissenschaftlerinnen wie etwa Irena Medjedović untersuchen vor allem die pragmatischen Probleme von Zweitauswertungen: Wie können mündliche Zeugnisse aus anderen Projektkontexten für neue Fragestellungen und Erkenntnisinteressen, für Reinterpretationen und andere disziplinäre Herangehensweisen zugänglich gemacht werden? ${ }^{12}$ Inwiefern und in welchem Maße behindert die Kontextgebundenheit des erhobenen Materials eine neuerliche Analyse? Im Zentrum steht der reflektierte Umgang mit der sekundären Auswertung von Interviews, nicht zuletzt, weil die Interviewbestände in den letzten rund 40 Jahren stark angewachsen sind und Sammlungswegweiser wie Methodenleitfäden nötig sind, um sich im Dickicht der Daten nicht zu verlieren.

Dass die geschichtswissenschaftliche Reflexion über Fragen der Sekundärauswertung erst spät einsetzte, eigentlich erst in den letzten Jahren, sei deswegen bemerkenswert, so Linde Apel, weil „seit Jahren das ,Ende der Zeitzeugen' proklamiert und der Zeitzeuge als mediale Figur historisiert" werde, "ohne dass sich das bisher auf die Historisierung der mündlichen Quellen ausgewirkt" habe. ${ }^{13}$ Mittlerweile aber liegen erste geschichtswissenschaftliche Studien und Forschungskooperationen vor, die sich zunächst ebenfalls praktischen Fragen der Archivierung und Sammlung von Interviews widmen. Erste Online-Editionen machen in den letzten Jahrzehnten aufgezeichnete Interviews erneut hörbar und in ihrer auditiven Anmutung zugänglich - und damit auch für die Sound History interessant, die sich indes weit häufiger den Geräuschen der Großstadt als den Erzählungen von Menschen widmet. ${ }^{14}$ Erinnert sei hier nur an das Online-Archiv der Interviews mit fast 600 Zwangsarbeitern und Zwangsarbeiterinnen, die im Zusammenhang ihrer Entschädigung in den 200oer Jahren von einem Team um Alexander von Plato geführt wurden. ${ }^{15} \mathrm{Ge}-$ plant ist die Edition einer Auswahl der von dem Soziologen David P. Boder schon 1946 geführten Interviews mit Überlebenden der Konzentrationslager, die so anders klingen als die uns heute auf eine geradezu bequeme Weise vertrauten mündlichen survivor-Zeugnisse. Sie irritieren unsere Hörgewohnheiten schon deswegen, weil es ganz junge Stimmen sind, die vom gerade überstandenen, noch ganz gegenwärtigen und vor allem alltäglichen Horror in den Lagern

\footnotetext{
12 Vgl. hier nur Medjedović/Witzel (2010); Medjedović (2014).

13 Vgl. Apel (2015), $243 \mathrm{f}$.

14 Vgl. Morat (2015); als Kompendium zur Sound-Geschichte vgl. Paul/Schock (2014).

15 http://www.zwangsarbeit-archiv.de/ [1.7.2017].
} 
erzählen. ${ }^{16}$ Und auch die LUSIR-Interviews werden unter der Leitung von Almut Leh digitalisiert und demnächst in einer Auswahl auf den Seiten des Archivs „Deutsches Gedächtnis“ wieder zu hören sein. ${ }^{17}$

Dass lebensgeschichtliche Sammlungen archiviert und Hördokumente erneut bereitgestellt werden, stellt die Voraussetzung einer breiteren, auch inhaltlichen Historisierung der Oral History dar. Hier setzt mein Versuch an, LUSIR als Pionierprojekt der bundesrepublikanischen Interviewforschung wissens- und historiografiegeschichtlich einzuordnen, auch wenn im Folgenden nur erste Möglichkeiten einer solchen Untersuchung ausgetestet werden können. Zum neu entstehenden Forschungszweig der Sekundäranalyse sollte wesentlich gehören, an einer History of Oral History im 20. Jahrhundert zu arbeiten, die durchaus auch transnational ausgeweitet werden kann und gerade mit Blick auf die international eng kooperierenden Netzwerke der Oral History auch muss. ${ }^{18}$ Wissens- und wissenschaftsgeschichtlich ist zu fragen, wie auf dem Weg der Interviewforschung innovatives Wissen generiert wurde, das auf produktive Weise über das allgemein Bekannte hinausging. Was machte in der ersten Hälfte der achtziger Jahre die innovative Spezifik der Oral History im Vergleich zu anderen Quellensorten aus? Aber auch: Wo lagen die Grenzen der Methode?

\section{Politische Ernüchterung und Idealisierung der Arbeiterklasse: Erwartungshorizonte}

Dass die LUSIR-Forscher und -Forscherinnen so grundlegend irritiert von den Begegnungen mit Arbeitern und Arbeiterinnen im Ruhrgebiet waren, lag zum einen an ihren eigenen politischen Vorerfahrungen, zum anderen an ihrem Vorwissen über Arbeiter, Arbeiterbewegung und Arbeiterklasse - beides hing eng miteinander zusammen. Am Anfang des Projekts hatten, so beschrieb Niethammer die kollektiven Erwartungen der Forschergruppe, „die meisten von uns geglaubt, daß unsere Wertvorstellungen und diejenigen unserer Befragten trotz unserer unterschiedlichen Sozialisation viele Gemeinsamkeiten hätten; und außerdem wussten wir, daß die Arbeiterklasse des Ruhrgebiets

16 Das Projekt wird von Axel Doßmann geleitet. Die über 100 Interviews sind auch jetzt schon, allerdings weitgehend unkommentiert, zu hören und nachzulesen: http://voices .iit.edu/[1.7.2017].

17 Vgl. https://www.fernuni-hagen.de/geschichteundbiographie/deutschesgedaechtnis/ [1.7.2017]; Leh (2015).

18 Vgl. Leo/Maubach (2013). 
eben nicht jener Teil des Volkes war, der Hitler an die Macht gebracht hatte“. ${ }^{19}$ Tatsächlich hatten die Stimmen für die NSDAP in den entscheidenden Wahlen 1932/33 im Ruhrgebiet weit unter dem Zustimmungsniveau anderer Regionen wie etwa Thüringens gelegen.

Bis auf Lutz Niethammer und Alexander von Plato, die 1939 und 1942 geboren worden waren, gehörten alle Mitglieder der Forschungsgruppe Jahrgängen der späten vierziger und frühen fünfziger Jahre an. Als „Flakhelfer der 68er“ standen sie politisch mehr oder weniger weit links. ${ }^{20}$ Um 1980 hatte jedoch bereits ein Prozess der Entradikalisierung eingesetzt, der sich auch auf der Ebene des internationalen Oral-History-Netzwerks nachvollziehen lässt, das sich etwa zur gleichen Zeit konstituierte. Alexander von Plato hatte dem maoistischen Flügel der Kommunisten, der KPD/AO, den Rücken gekehrt, Detlev Peukert, der das Projekt mit konzipiert hatte, dann aber weder Interviews führte noch Texte schrieb, sondern ein Jahr vor dem ersten LUSIR-Band sein Buch Volksgenossen und Gemeinschaftsfremde herausbrachte, war Ende der siebziger Jahre aus der DKP ausgetreten. ${ }^{21}$ Der Glaube an linke Utopien war ein gutes Dutzend Jahre nach der Studentenrevolte und dem Gesellschaftswandel um 68 deutlich angeknackst. ${ }^{22}$ In einem Text über Peukert hat Ulrich Herbert aus der Rückschau nachgezeichnet, warum die Arbeiter für die jungen Intellektuellen genau zu diesem Zeitpunkt ihre Bedeutung als „identifikationsfähige Subjekte" verloren hatten. Erstens sei die Arbeiterschaft nicht nur in der Bundesrepublik, sondern auch in Frankreich oder Italien um und nach '68 alles andere als "ein Element antikapitalistischen Furors“ gewesen. ${ }^{23}$ Zweitens sei die „klassische Industriegesellschaft mit Schwerindustrie und ungelernten Massenarbeitern“ in der Phase zwischen 1975 und 1985 „unübersehbar“ an „ihr Ende" gekommen.

Der Boden für die Erkenntnis, dass die Arbeiterschaft im Nationalsozialismus von den Vorteilen, die etwa die nationalsozialistische Sozialpolitik für sie bereitgehalten hatte, durchaus korrumpiert worden war, war also längst bereitet. Gleichwohl hatten die Mitglieder von LUSIR, als sie auszogen, die Erfahrungen der Ruhrgebietsarbeiter zu erkunden, zweifellos noch emphatische Vorstellungen von ihrem Gegenüber, vor allem von der Opposition der Arbeiterklasse gegen den deutschen „Faschismus“. Detlev Peukert etwa hatte selbst Widerstandsforschung betrieben und schon 1976, 26 Jahre alt, seine

\footnotetext{
19 Niethammer (1986a), S. $21 \mathrm{f}$.

20 Der Begriff stammt aus der Autobiografie von Raulff (2014), S. 11.

21 Vgl. Peukert (1982).

22 Einen solchen Entradikalisierungsprozess beschreibt eindringlich Lethen (2012).

23 Dieses und das folgende Zitat: Herbert (2015), S. 42.
} 
Staatsexamensarbeit Ruhrarbeiter gegen den Faschismus vorgelegt, in der er die dogmatische Dichotomie von Monopolkapital und Arbeiterschaft noch weitgehend reproduzierte. ${ }^{24}$ In den Interviews mit den Arbeitern und Angestellten der Ruhrzechen und Industriebetriebe wurde nicht zuletzt die starre Vorstellung von der Arbeiterschaft als einer kollektiven politischen Kraft radikal individualisiert und relativiert - und diese relativierende Funktion von LUSIR ist vielleicht ein Grund unter anderen dafür, dass Peukert recht schnell aus dem Projekt ausschied.

\section{Lebensgeschichte und Sozialkultur: das ganze Arbeiterleben}

Der gerade geschilderte Prozess der politischen Ernüchterung bis Anfang der achtziger Jahre setzte sich auf der Ebene der Forschung fort und wurde durch die empirischen Ergebnisse, die LUSIR bereithielt, verstärkt und in einen wissenschaftlichen Differenzierungsprozess übersetzt. In dem Maße, wie sich der Forschungsfokus auf das ganze Arbeiterleben richtete und nicht nur auf die dramatischen Ausschnitte des politischen Engagements von Aktivisten der Arbeiterbewegung, verlor der politische (Klassen-)Kampf an Bedeutung und Relevanz. So wenig aufregend die beiden Titelbegriffe - Lebensgeschichte und Sozialkultur - klangen und klingen, so sehr deuten sie die Befunde von LUSIR schon an: Betrachtete und untersuchte man die ganze Lebensgeschichte von Arbeitern im breiten Umfeld ihrer Alltags- und Sozialkultur, dann stürzten Großerzählungen wie die Klassenkampf- oder Widerstandsgeschichte in sich zusammen. Unterhalb der Ebene ideologischen Kampfes und politischen Engagements stießen die Oral Historians auf ernüchternde Formen eines zunehmend entpolitisierten Arbeiterlebens und auf breite Zonen des Konsenses mit dem NS-Regime.

Was die Interviews zutage förderten, war eine andere Arbeitergeschichte in der ersten Hälfte des 20. Jahrhunderts, nicht politisch und progressiv, sondern individualistisch und resignativ. Sie erzählte nicht mehr von der politischen Mobilisierung der Sozialdemokratie und vor allem der Kommunisten in der Weimarer Republik, von der Spaltung und Zerschlagung der organisierten Arbeiterbewegung durch das nationalsozialistische Terrorregime in der Phase der "Machtergreifung“, nicht mehr von Opposition und Widerstand gegen die Diktatur, sondern von den kleinen und größeren Anpassungsprozessen im nationalsozialistischen Arbeiteralltag, die den Einzelnen zu einem besseren

24 Vgl. Peukert (1976). Die Dissertation war bereits moderater; vgl. Peukert (1980), dazu Hachtmann/Reichardt (2015); Wachsmann (2015). 
Leben verhalfen. Denkbar nüchtern formulierte Ulrich Herbert diese Einsicht in seinem zusammenfassenden Beitrag am Anfang des dritten Bandes von LUSIR: „Zunächst zeigten die Erinnerungen unserer Interviewpartner, daß die dramatische politische Entwicklung im Ruhrgebiet während der Weimarer Zeit, der hohe Mobilisierungsgrad der Arbeiterparteien oder die starke Stellung der Kommunisten falsche Vorstellungen von der Bedeutung von >Politik im Leben der Arbeiterschaft hervorruft.“25 Wenn „Politik“ „Parteipolitik, Programmatik, Organisationsfragen, aktive Beteiligung oder Diskussion über politische Themen“ meine, dann habe sie „im Leben der meisten unserer Interviewpartner auch zu dieser Zeit nur eine geringe Rolle und bei nicht wenigen sogar gar keine" gespielt. So spiegelte sich in der Geschichte der vormaligen Identifikationsobjekte - auch, aber nicht nur - die Zeitdiagnose der achtziger Jahre: der Prozess einer unpolitischen, auf privaten Wohlstand und persönliches Fortkommen ausgerichteten Individualisierung, die Atomisierung der Gesellschaft, die geplatzten Träume der Post-68er.

Es müssten genauer untersucht werden, inwiefern und wie die wissenschaftlichen Befunde mit dem zeitgenössischen Blick auf die Gesellschaft der Bundesrepublik zusammenhingen. Vor allem aber resultierten sie aus der methodologischen Entscheidung, die ganze Lebensgeschichte der Interviewpartner zu erfragen und nicht, wie vor den Methodenrevisionen eines Fritz Schütze in der qualitativen Sozialforschung üblich, nur themenzentrierte Ausschnitte entlang standardisierter Fragenkataloge. Vermutlich hatte diese Entscheidung für eine umfassende Perspektive auch etwas mit der Wendung auf das Subjekt in den Post-68er-Milieus der siebziger und achtziger Jahre zu tun; die Interviewpartner sollten nicht für fremde wissenschaftliche Erkenntnisinteressen instrumentalisiert, sondern zu ihrer eigenen Geschichte befreit und in ihrer individuellen Einzigartigkeit wahrgenommen werden. Darum orientierte sich die frühe bundesdeutsche Oral History um Lutz Niethammer zunächst lose an der Interviewmethode Fritz Schütze, der das themenzentrierte Interview bereits in den siebziger Jahren zur Erfragung umfassender biografischer Narrative hin geöffnet hatte. Schütze wollte herausfinden, welchen Verlaufstypen Lebenserzählungen folgten, wenn sie sich nach den eigenen, immanenten Relevanzkriterien und ungestört von den externen Frageinteressen der Wissenschaftler entfalten konnten. Primär interessierten ihn deswegen die Konstruktionsmechanismen von Erinnerungen in der Erzählgegenwart. ${ }^{26}$

Daraus ergeben sich bereits die entscheidenden, von Niethammer stets betonten Unterschiede zur sozialwissenschaftlichen Interviewforschung nach

25 Dieses und das nächste Zitat Herbert (1985), S. 21.

26 Vgl. Schütze $(1978,1981)$. 
Schütze: Erstens ging das LUSIR-Team davon aus, dass gerade die Interaktion zwischen den Gesprächspartnern eine erkenntnisfördernde Kraft besaß, während Schütze vom Interviewer erwartete, dass er sich wie unsichtbar im Hintergrund des Gesprächs halten sollte, damit die Erinnerungserzählung ungestört Gestalt annehmen konnte. Auf den Begriff gebracht und wirklich elaboriert wurde dieser Methodenunterschied aber erst im Laufe der wenige Jahre später folgenden Interviewtour durch die DDR; nun sprach Niethammer, der in der DDR auch selbst Interviews führte, ausdrücklich davon, „Gedächtnisspuren neu zu eröffnen und die Erinnerung zu erweitern“machte also das explizite thematische Umsteuern gegen die Erinnerungsrichtung der Interviewten zu einem festen Bestandteil der Interviewführung. ${ }^{27}$ Aus der Rückschau erklärt Niethammer diese Entscheidung für eine „hochgradig dialogisch[e] Aktivität zur Optimierung der Gedächtnisleistungen“ mit dem generellen „Misstrauen gegenüber allen Einzelnen“ in posttotalitären Gesellschaften, deren Erinnerungen durch eine stets mitlaufende, historische Quellenkritik in the making überprüft werden sollten. ${ }^{28}$ Auf diesem methodischen Weg wollte das LUSIR-Team, zweitens, die verschiedenen Zeitschichten, die die Interviewtexte enthielten, isolieren und rekonstruieren. Dieser methodisch kontrollierte Prozess der Annäherung an die vergangene Erfahrung war für Sozialwissenschaftler hingegen weder sonderlich interessant, noch glaubten sie an die Möglichkeit einer Erfahrungsrekonstruktion qua Erinnerungsinterview. Ihnen ging es, wie Niethammer in Fragen - Antworten - Fragen formulierte, „am wenigsten“ um den Inhalt der Erinnerungen, sondern „vielmehr um die Prägung ihrer aktuellen Zurichtung durch die umgebende Gesellschaft“. ${ }^{29}$

Trotz dieser unterschiedlichen Erkenntnisinteressen war beiden Ansätzen das Interesse am ganzen Leben gemein, sodass die Interviewer in LUSIR ebenfalls einen radikal offenen Erzählimpuls an den Anfang jeden Interviews setzten: „Bitte erzählen Sie Ihr Leben!“ In den folgenden, freien Lebensbericht sollten die Interviewer (jenseits von Sachnachfragen) möglichst nicht eingreifen, um ihre Gesprächspartner nicht aus dem Konzept ihrer Selbstbiografisierung zu bringen, und tatsächlich reagierte manch ein ehemaliger Arbeiter in LUSIR ungehalten, wenn neugierige Nachfragen ihn beim Erinnern störten. Üblicherweise endete der freie Lebensbericht in einer Art kulminierendem

27 Niethammer (1991), S. 29. Im zentralen Methodentext Fragen - Antworten - Fragen finden sich Begriff und Methodenschritt noch nicht, allerdings der Gedanke von der zentralen Bedeutung einer mitsteuernden, nicht nur affirmativen, immanenten Interviewführung. Niethammer (2013), S. 303. S. auch das jüngst veröffentlichte Interview: Oral History in der deutschen Zeitgeschichte (2017), v.a. S. 120-125. Vgl. zur Methode in der formativen Phase der Oral History Maubach (2013). 
Erschöpfungszustand - vornehm wissenschaftlich „Kataklysmus“ genannt -, dann geriet der Erinnerungsfluss ins Stocken und die Interviewten baten um Nachfragen.

Die LUSIR-Mitarbeiter lauschten den Lebensgeschichten, wie erwähnt, nicht mit einem soziologischen, gewissermaßen synchronen Interesse daran, wie biografische Erzählungen sich in der Erzählgegenwart verfertigten, sondern mit einem genuin historischen Interesse (das der heutigen historischen Interviewforschung, die lebensgeschichtliche Narrative in der Regel ebenfalls als Gegenwartskonstrukte begreift, in vielen Fällen verlorengegangen ist). Sie wollten etwas über die Erfahrungsrealität der Arbeiter und Angestellten im Revier vor, im und nach dem Nationalsozialismus erfahren, und als zwar ernüchterte, aber immer noch hoffnungsbereite Linke erwarteten sie Erzählungen von ideologischer Distanz zum Nationalsozialismus, wenn nicht von Alltagsresistenz oder gar Opposition gegen die Diktatur. Umso mehr überraschte sie der frappierende Kontinuitätswille, mit dem viele ehemalige Arbeiter die entscheidende politische Zäsur 1933 einfach übersprangen. Die nationalsozialistische Machtübernahme am 30. Januar 1933, zugleich das traumatische Symboldatum der folgenden, systematischen und äußerst gewalttätigen Zerschlagung der organisierten Arbeiterbewegung, wurde in den Lebenserzählungen entweder ganz übergangen oder nur abstrakt - wie ein Datum aus einem Schulbuch - memoriert. Die Frage, was sich am Leben der Arbeiter und Angestellten nach 1933 verändert habe, drängte sich also auf. Ulrich Herbert stellte sie einem seiner Interviewpartner, dem 1908 geborenen Krupp-Angestellten Heinrich Bergmann, in jedem einzelnen der insgesamt drei Interviews, die er mit ihm führte, und im allerletzten, Anfang 1982 geführten Gespräch gleich zwei Mal. Bergmann aber blieb unerschütterlich und gab ebenso stoisch wie vorsichtig immer wieder dieselbe Antwort: „Gar nix.“

U.H.: Was hat sich denn so bei Krupp, ver-... im Betrieb... Was hat's verändert, oder verändert dadurch, dass die Nazis an der Macht waren?

H.B.: Gar nix.

U.H.: Ich mein so die, die, der Aufbau...

H.B.: Ich bin in einem Betrieb...

U.H.: Der Obmann oder die...

H.B.: Ich bin in einem Betrieb gewesen, in dem hauptsächlich Fachleute beschäftigt waren. Und da waren alles alte Leute, so alt wie mein Vatter, so ungefähr, alles die, die waren darin beschäftigt, also alte Gewerkschaftler und alle sowas, junge Leute waren recht wenig dadrin, nich. ${ }^{30}$

30 Drittes Interview Ulrich Herbert mit Heinrich Bergmann (Pseud.) vom 24.2.1982, 4/4:20-29. 
Es hatte sich nichts verändert, erklärte Bergmann dem aus dem Konzept seiner Vorannahmen gebrachten jungen Ulrich Herbert, weil die alten, erfahrenen Facharbeiter - lauter politisch aufrechte, ehemalige Gewerkschafter - sich dem politischen Zugriff dadurch hätten entziehen können, dass sie nicht zu ersetzen und unabkömmlich gewesen seien. Die meist jungen, un(aus)gebildeten Nazis hingegen seien, wie Bergmann fortfuhr, in Arbeitsbereichen untergekommen, in denen sie nur einfache Hilfs- und Handlangertätigkeiten hätten verrichten müssen. Bergmann verlieh seiner Erzählung Plausibilität, indem er auf zwei miteinander verbundene Argumente zurückgriff: berufsspezifische und generationelle. Seine Erklärung schloss aus, dass versierte und verdiente Facharbeiter - oder Angestellte mit großer Expertise, so wie er - überhaupt Nazi sein oder werden oder sich auch nur zeitweise vom Nationalsozialismus hätten korrumpieren lassen können.

Gerade aber die Tatsache, dass die Interviewpartner die politische Zäsur 1933 übersprangen und die "guten Jahre“ ab etwa 1935 nur auf Nachfrage schilderten, war Hinweis auf eine mindestens partielle Annäherung an den Nationalsozialismus. Dass Ulrich Herbert seine Frage während der drei Interviews in Variationen und aus unterschiedlichen Richtungen immer wieder stellte, macht nicht nur seine Irritation deutlich, sondern zugleich, dass er Fährte aufgenommen hatte und Erkenntnis witterte. Und tatsächlich entstand aus dieser und anderen, ähnlichen Interviewpassagen eine Interpretation und sogar eine spezifisch erfahrungsgeschichtliche Periodisierung, die sich nicht an politischen Großzäsuren, sondern an den kleinen Einschnitten im persönlichen Leben des Einzelnen orientierte. Politische und individuelle Periodisierung waren "nicht kongruent“". ${ }^{31}$ Aber wie unterteilten die Interviewpartner ihr Leben in Abschnitte?

Die Arbeiter und Arbeiterinnen sowie die kleinen und mittleren Angestellten, zu denen Bergmann gehörte, stellten eine ganz eigene Zeitrechnung auf. Was ihr Leben in Phasen teilte, so legte Ulrich Herbert in seinem Aufsatz zur lebensgeschichtlichen Periodisierung im ersten LUSIR-Band dar, waren nicht die großen Wellen der politischen (Klassenkampf-)Geschichte, sondern die kabbelige See der guten und der schlechten Zeiten. ${ }^{32}$ Gute Zeiten waren Zeiten sicherer Arbeitsplätze und eines bescheidenen Fortkommens im überschaubaren Rahmen des eigenen Lebens. Deswegen waren gute Zeiten immer auch ruhige Zeiten - abseits, ja jenseits von Revolution und politischem Kampf. Die schlechten Zeiten waren die Jahre der Massenarbeitslosigkeit und Armut, die

31 Herbert (1985), S. 24.

32 Vgl. Herbert (1986a). 
die Weimarer Jahre prägten und über das Jahr 1933 hinweg reichten. Erst danach, um 1935, als die Arbeitsplätze wieder sicher waren, ging es bergauf.

Was die LUSIR-Forscher herausfanden, provozierte - nicht zuletzt die Forscher selbst - und korrespondierte mit der eigenen politischen Ernüchterungserfahrung. Manchmal hörte es sich fast an, als gäben die Ruhrarbeiter NS-Propaganda zum Besten. Sie verbanden die Goldenen Dreißiger mit der „neuartige[n] Erfahrung eines über Jahre stabilen Arbeitsplatzes“, ${ }^{33}$ während es die Goldenen Zwanziger gar nicht gab, weil kürzere oder längere Phasen der Arbeitslosigkeit die gesamte Zeit der Weimarer Republik perforierten und die Erinnerung dunkel tönten. Arbeitsplatzsicherheit infolge von Militarisierung und Aufrüstung war aber nicht der einzige Faktor, der die Erinnerung noch fünfzig Jahre später vergoldete. Hinzu kam die „sozialpolitische Offensive der Nationalsozialisten", die vielen Arbeiter- und Angestelltenfamilien im Ruhrgebiet einen langsam steigenden Lebensstandard brachte: Sichtbar wurde das beispielsweise an der zunehmend besseren, beispielsweise über ein Ehestandsdarlehen finanzierten Ausstattung des Arbeiterkottens, und gelegentliche kleine Urlaubsreisen, damals in diesen Schichten noch ganz unbekannt, waren als Erholung körperlich und seelisch spürbar. ${ }^{34}$ Erst als der Krieg - in der Regel spät, weil in den Rüstungsbetrieben an der Ruhr viele Arbeiter und Angestellte unabkömmlich gestellt wurden - in das eigene Leben einbrach, spätestens 1943 mit den alliierten Flächenbombardements, wurden die Zeiten wieder schlecht, eine Erfahrung, die über das Kriegsende hinausreichte. In den Wirtschaftswunderjahren konnte dann wieder an die guten Dreißiger angeschlossen werden: Der Habitus der angestelltenhaften Kruppianer der fünfziger Jahre, die, wie Michael Zimmermann sarkastisch schrieb, in Anzug und mit Schlips zur Arbeit gingen und ihre Kaffeepulle nicht mehr offen und arbeiterstolz auf dem Rücken trugen, sondern voller Scham in ihren Aktentaschen verbargen, hatte seinen Ursprung im Nationalsozialismus. ${ }^{35}$

Dieser Wechsel von guten und schlechten Zeiten, der die lineare Fortschrittszeitrechnung der Arbeiterbewegung durch den Kreislauf im realen Kapitalismus ersetzte, in dem Hausse und Baisse konjunkturbedingt abwechseln, war indes nur sichtbar geworden, weil die LUSIR-Forscher das ganze Leben ihrer Protagonisten in den Blick nahmen. Dabei sollten die Lebensgeschichten in den breiten Kontext der Sozialkultur platziert werden - das war der titelgebende Zusammenhang. Sozialkultur, wie die LUSIR-Mitarbeiter

\footnotetext{
33 Herbert (1985), S. 25.

34 Zum Einsatz des Ehestandsdarlehens vgl. Einfeldt (1986), S. 274/275; zur Bedeutung des Urlaubs an sich vgl. Herbert (1985), S. 27.

35 Vgl. Zimmermann (1986), S. 109, 113.
} 
sie verstanden, umfasste weit mehr als das klassische Arbeitermilieu: Nachbarschaftsbeziehungen, Geschlechterverhältnis und Familienbande, Vereinsleben und Betriebsgemeinschaften, Kulturerlebnisse und Reisen. Genau definiert und einheitlich verwendet wurde der Begriff in den Beiträgen nicht, manchmal taucht als Synonym der aus der Phänomenologie und Wissenssoziologie und ursprünglich von Alfred Schütz stammende Begriff der „Lebenswelt“ auf. Aber auch wenn die Begriffsverwendung nicht vollends reflektiert wurde und insgesamt fluide blieb, erwies sich der Versuch, Arbeiterleben jenseits ihres klassischen Milieus in breiteren gesellschaftlichen Zusammenhängen zu untersuchen, als produktiv. Die Trennlinien zwischen den Milieus waren schon in der Weimarer Zeit viel durchlässiger als gedacht, wie Bernd Parisius aus seinem Interview mit dem Verwaltungsangestellten Josef Paul lernte. Paul erzählte, dass sein Vater, ein Sozialdemokrat, einen Arbeiterverein gegründet habe; die Nachfrage des Interviewers diente zunächst nur dem Verständnis:

B.P.: Was war das für ein Arbeiterverein?

J.P.: Das war der katholische Arbeiterverein St. Mariä Himmelfahrt. Den hat mein Vater mitgegründet.

B.P. (irritiert): Ach so, denn war er aber trotzdem SPD-Wähler?

J.P.: Ja, ist er trotzdem gewesen.

B.P. (hoffnungsvoll): Hm. Aber nicht organisiert in der SPD?

J.P.: Doch, er war organisiert in der SPD.

B.P.: Ach so.

J.P.: Er war organisiert in der SPD, weil mein Großvater war ein SPD-Mann.

(Pause)

B.P.: Ach so, ich hab immer gedacht, daß so'n katholischer Arbeitervereinen und die SPD sich...

J.P: Nein, das hat sich vertragen. ${ }^{36}$

Dass sich das Arbeitermilieu in der Erzählung Josef Pauls so unspektakulär auflöste, rief beim Interviewer hörbar Irritationen hervor, die Alexander von Plato in seinem Aufsatz „Ich bin mit allen gut ausgekommen“ in eine Interpretation übersetzte. Untersuchte man die Ruhrarbeiterschaft „von unten“, in ihrer Lebenswelt, dann verlöre sie ihre „scharfen Konturen“, eine produktive „Unschärferelation“, die dazu führte, dass die Arbeiterklasse am Ende nicht mehr existierte. ${ }^{37}$ Die damals meist noch im Kollektiv untersuchte

36 Zitiert bei von Plato (1986), S. 42 f. und von mir neu transkribiert: 2/31:52-32:18.

37 Von Plato (1986), S. 59. 
Arbeiterklasse verlor ihren Zusammenhalt, war zwischen 1930 und 196o immer weniger als eine historisch und politisch distinkte Gruppe zu erkennen und löste sich in die Gesellschaft hinein auf.

Dieser Auflösungsprozess, durch die gewaltsame Zerschlagung der Arbeiterbewegung nach 1933 forciert und vollendet, wurde aber nicht nur als Verlust erinnert, sondern viel häufiger als ein Zugewinn an sozialer Mobilität und Macht erzählt. Dass gerade die nationalsozialistische Sozialpolitik und die Politik der Aufrüstung und Militarisierung vielen der Befragten zu Erfahrungen von sozialem Aufstieg und persönlicher Macht verholfen hatten, war am Anfang der achtziger Jahre freilich kaum sagbar - aber vielen noch erinnerlich. Weil diese Erfahrungen individueller Expansion für die Interviewten sinnstiftend und also von großer Bedeutung waren, bahnten sie sich trotzdem einen Weg ans Tageslicht der Erzählungen und brachen sich in harmlosen kleinen Alltagsgeschichten Bahn.

\section{Geschichten von sozialem Aufstieg und persönlicher Macht}

Eine der epistemologisch wichtigsten Erkenntnisse in LUSIR war, wie erwähnt, dass die ins Mikrofon gesprochenen Lebensgeschichten nicht nur gegenwärtigen Identitätskonstruktionen und Erzählkonventionen gehorchten, also zwar in actu verfertigt wurden, aber - wie Goldstücke im sandigen Sieb authentische Erfahrungen enthielten. Ein wesentliches Verfahren zur deren „Beglaubigung“ bestand darin, „spontane Erzählungen“ ausfindig zu machen, wie Lutz Niethammer in der Einleitung zum ersten Band noch tentativ und im Methodenaufsatz „Fragen - Antworten - Fragen“ am Ende des dritten Bandes programmatisch formulierte. Solche „Erzählungen in der Erzählung des Interviews" konnten verschiedenen Zeitschichten angehören: Manchmal hatten sie sich zu oft erzählten Geschichten verfestigt, manchmal aber waren sie noch nicht durch den Filter bewusster Reflexion gegangen, sondern wurden wie ein stream of consciousness - ,frei von der Leber weg' erinnert. Nicht selten widersprachen sie dann den intensiver choreografierten Erzählteilen, also etwa programmatisch verkündeten politischen Einstellungen, moralischen Werturteilen oder den literarisierten, immer wieder zum Besten gegebenen und in der Wiederholung längst gegenwärtigen Sinnerfordernissen angepassten Anekdoten. Insgesamt gingen die einzelnen narrativen Elemente eines lebensgeschichtlichen Interviews eben nicht in einer glatten Sinnkonstruktion auf, sondern standen im Widerspruch zueinander oder hinterließen Bedeutungslücken - und gerade die Brüche, die im Erzählen entstanden, waren interessant und wiesen, wie die Titel der LUSIR-Bände, auf Zeitsprünge hin. 
Die Erinnerungserzählungen bargen also immer dann authentische Erfahrung, wenn eine Person sich unwillkürlich, ja geradezu körperlich reflexhaft erinnerte, im Duktus von Und-dann-Erzählungen, in denen die Chronologie der Erlebnisse noch nicht sinnhaft geordnet war, sondern nur nacherzählt wurde; als eine bunte und plastische Chronik des Erlebens und nicht als pointenreiche Anekdote mit einer "Moral von der Geschichte“ am Ende. Ein Leitbeispiel für Niethammer war das Interview mit dem eingangs erwähnten Metallarbeiter Fritz Harenberg, der sich an den „Blitzkrieg“ als Beutezug erinnerte. Von ihm stammte auch der Satz, der dem ersten Band seinen verwirrend schönen Titel gab: „Die Jahre weiß man nicht, wo man die heute hinsetzen soll.“ Harenberg war nicht sehr eloquent, auch nicht besonders gebildet. Gerade deswegen war seine Erinnerung an den Blitzkrieg eine „bunte Burleske“, die den Erlebnissen chronologisch folgte und von den reihenweise in die Straßen eingebauten Bordellen in Nancy über die Schokoladengeschäfte in Sarajewo bis hin zum dortigen jüdischen Friedhof reichte; diesen hatte die Gestapo auf der Suche nach Reichtümern durch einige jüdische Bewohner der Stadt aufgraben lassen. Die Aussage, die diese Chronik des Wohllebens im Krieg beschloss, war alles andere als ein reflektiertes Resümee: Wie eine „Made im Speck“ habe er gelebt. ${ }^{38}$

Solche unwillkürlich erzählten Geschichten lagen weit von den programmatischen Selbstverortungen entfernt, in denen sich die Interviewpartner weiterhin als Teil einer solidarischen, zum Nationalsozialismus auf Abstand gebliebenen Arbeiterklasse stilisierten. Sie zeigten vielmehr, dass und wie sehr auch diese soziale Schicht von der nationalsozialistischen Sozial- und nach 1939 von der Eroberungspolitik im Krieg korrumpiert worden war. Die "guten Jahre" während der NS-Zeit ermöglichten den Interviewpartnern Expansionen in Lebensbereiche, die ihnen zuvor verschlossen geblieben waren. Genau diese positiven Erfahrungen individueller Expansion verlangten noch fünfzig Jahre später nach Ausdruck. Wollte man sie analytisch unterscheiden und grob typisieren, handelte es sich erstens um Expansionen über die Grenze der eigenen Schicht, also um Verbürgerlichungserfahrungen, zweitens um Erfahrungen räumlicher Mobilität und drittens um (berufliche wie persönliche) Überordnungs- und Machterfahrungen.

Um dies am Beispiel zu erläutern, nehme ich Heinrich Bergmanns Lebensgeschichte wieder auf. Für ihn war die Kontinuitätskonstruktion über das Jahr 1933 hinaus eine wesentliche Voraussetzung, um seinen Erfahrungsexpansionen zum Ausdruck verhelfen zu können. Im Zusammenhang der wiederholten Nachfragen des Interviewers Ulrich Herbert, was sich nach 1933 verändert habe, erzählte er, dass ihm von den Kollegen seiner Abteilung - alle „keine 
Nazis“, sondern verdiente Facharbeiter - die Zuständigkeit für Kraft durch Freude übertragen worden sei, also für jene sozialpolitische Initiative der Nationalsozialisten, die darauf zielte, auch den einfachen Volksgenossen (im Tausch für Systemloyalität) Reise- und Kulturerlebnisse zu ermöglichen, und die als steingewordene und Torso gebliebene gigantische Freizeitarchitektur in Prora auf Rügen bis heute besichtigt werden kann. Fortan musste Bergmann „Reisen vermitteln“ und „Reisen aufnehmen, wenn einer weg wollte“ - "genau wie beim Reisebüro heute“, wie er erklärte. ${ }^{39}$ Über einige Minuten hinweg und mit deutlicher Begeisterung in der Stimme stellte Bergmann minutiös sein nationalsozialistisches Reisebüro vor, das er „im Nebenberuf“ betrieben habe. Weil er selbst einige Fahrten etwa in den Harz mitgemacht hatte, habe er den Arbeitskollegen gegenüber glaubhaft die Werbetrommel rühren können. Außerdem verkaufte er für kleines Geld Opern- und Theaterkarten, und weil nicht alle abgeholt wurden oder einzelne übrig blieben, verschaffte ihm die kleine Machtposition bei der Kartenverteilung selbst das Privileg häufiger Opern-, Operetten- und Theaterbesuche; so kam Bergmann in den ganz bildungsbürgerlichen Genuss von Lessings Schauspiel Emilia Galotti. So oft „wie zu der Zeit“, bilanzierte er, sei er "nachher und vorher nich' im Theater gewesen“. Der soziale Aufstieg, die Privilegierung und Verbürgerlichung, die schon daran sichtbar wurden, dass Bergmann den bürgerlichen Dresscode einhielt und seinen schwarzen Hochzeitsanzug nun regelmäßig ausführte, machten diese Erinnerungen für Bergmann noch Anfang der achtziger Jahre attraktiv und erzählenswert, auch wenn sie längst nicht mehr zeitgemäß und sogar irgendwie kompromittierend waren.

Ein sprachliches Mittel, um diesen kompromittierenden Erfahrungen Legitimität zu verleihen, waren nicht nur die großen, sondern auch die kleinen Kontinuitätssuggestionen. Was eigentlich nicht sagbar, weil nicht gesellschaftsfähig war, versuchte Bergmann für Ulrich Herbert - vergeblich, wie sich zeigen sollte - zeitgemäß zu übersetzen. So klassifizierte er seine KdF-Funktion harmlos als „Reisebüro“ oder nannte den „Vertrauensrat" bei Krupp umstandslos „Betriebsrat" und verwandelte auf diese Weise seine Handlangerdienste für die Nazis, die für das private Glück einer Nordlandfahrt mindestens die oberflächliche Loyalität ihrer „Volksgenossen“ einforderten, in ein ehrenamtliches Engagement für ein gutes Betriebsklima. Paradoxerweise reproduzierte er damit noch im Rückblick die für die Vertrauensräte propagandistisch und politisch ausersehene Funktion, eine „harmonische Betriebsgemeinschaft“ zu schaffen. Der gerade dreißigjährige Ulrich Herbert verfolgte staunend - und

39 Die folgenden Zitate im Interview von Ulrich Herbert mit Heinrich Bergmann (Pseud.) vom 24.2.1982, 4/07:0off. 
dann zunehmend verärgert -, wie Bergmanns Erzählungen in immer weitere Funktionsbereiche expandierten: Auch die „Freud- und Leidkasse“, aus der beispielsweise Hochzeitsgeschenke oder Trauerkränze bezahlt worden seien, habe er verwaltet und sei daneben für die finanzielle Verwaltung der Krupp'schen „Kameradschaftsabende“, die „revisionsfähig“ abgerechnet werden mussten, verantwortlich gewesen, eine Aufgabe, mit der der „Betriebsrat“, wie er stolz erzählte, überfordert gewesen sei:

U.H.: Betriebsrat hieß der aber nich' zu der Zeit, der hieß anders.

H.B.: Ja, Vertrauensrat.

U.H.: Vertrauensrat, ja.

H.B.: Vertrauensrat.

U.H.: War aber datselbe, meinen Sie?

H.B.: Ja, äh... funktionsmäßig datselbe... Bloß der Name is' anders. ${ }^{40}$

Die Erfahrung von beruflicher Verantwortung und persönlicher Macht inmitten eines nationalsozialistischen Vorzeigebetriebs - denn das war Krupp seit 1940 - war nur über die Suggestion eines im Grunde unverändert gebliebenen Betriebsalltags erzählbar. Dass diese kleinen sprachlichen Kontinuitätsfinten Herbert irritierten, weil er sie durchschaute, machen die höflich en passant gestellten, aber dennoch spitzen Nachfragen deutlich, die er sich besonders im letzten Interview Anfang Februar 1982 zugestand. Während er zuvor nur selten aus der Rolle des neutralen, höchstens Sachnachfragen stellenden Zuhörers gefallen war, nahm er sich jetzt das Recht zur ironisch-kritischen Randbemerkung.

Als die Erzählung von Bergmanns Funktionenfeldzug langsam ausgelaufen war und Herbert nach einem Aschenbecher verlangt und kurz geschwiegen hatte, hakte er ein - und jetzt sind das angestaute Befremden und all seine Ungeduld beim Hören der kaugummilangen, Anerkennung heischenden Erzählungen seines Gegenübers deutlich herauszuhören: „Was war denn nun eigentlich nationalsozialistisch an den Nationalsozialisten oder [war alles eigentlich] ganz normal, wie Sie das erzählen?"41 Bergmann verstand schnell, dass es jetzt galt, in ein zeitgemäßes Erinnern überzuwechseln, und hob seine kritische Distanz zum NS-Regime hervor. Man habe ja versucht, sich "so weit wie möglich vor allen diesen [nationalsozialistischen] Veranstaltungen“ zu drücken. Und zum Beweis seiner antifaschistischen Haltung erzählte er, vermutlich nicht zum ersten Mal, eine Anekdote vom Besuch Hitlers in seiner

40 Drittes Interview Ulrich Herbert mit Hermann Bergmann v. 24.2.1982, 4/13:36-28.

41 Ebd., 4/15:30-34. 
Abteilung. Nachdem die SS-Männer den „Adolf“ ins Büro geschoben hatten, hätten die Angestellten, so Bergmann, kaum aufgeschaut, geschweige denn sich zum Hitlergruß bequemt: „Ja, wat sollten wir sagen, wir konnten doch nich' >Heil Hitler< schreien, wir sollten doch arbeiten und nich' schreien, nich'? Bei uns war mucksmäuschenstill. ${ }^{42}$ Bergmanns Erzählung war erkennbar darauf angelegt, Harmonie und Übereinstimmung zwischen den Gesprächspartnern zu evozieren; sie war ein Friedensangebot.

Was ist wahr, was gelogen? Oder stimmt beides? Möglicherweise führen gerade die Widersprüche, die manch einer nach 1933 mit sich austragen musste: innerlich und schweigend - "mucksmäuschenstill“ - dagegen zu sein und gleichzeitig das Regime zu stützen, weil man von ihm profitierte, dicht an die vergangene Erfahrung heran. Jedenfalls fördern auch jüngere erfahrungs- und erinnerungsgeschichtlich perspektivierte Untersuchungen zur „Volksgemeinschaft" solche "dissonant lives" und kognitiven Spaltungen zutage..$^{43}$ Jenseits der ideologisch fanatisierten, radikalen Haupttäter scheint ebendiese Mischung aus innerer Distanz und äußerem Mittun der Normalfall einer Existenz in der Diktatur zu sein. Mit geläufigen wissenschaftlichen Dichotomien, die entweder Zwang oder Freiwilligkeit, Gewalt oder Affirmation betonen, sind sie nicht zu fassen. Lebensgeschichtliches Erinnern konnte das Widersprüchliche integrieren: die in Kontinuitätskonstruktionen versteckten Aufstiegs- und Machterfahrungen in den "guten Jahren“ und die zur Anekdote geronnene Behauptung einer antifaschistischen Distanz zum Regime.

\section{Erinnerungen - Anathema historischer Synthesen?}

Dass die Quellen, wie gezeigt, voller produktiver Widersprüche steckten, war eine Voraussetzung für die innovativen Erkenntnisse, zu denen LUSIR gelangte. Wohl zum ersten Mal wurden die Konturen der später so gründlich erforschten "Volksgemeinschaft" plastisch sichtbar und das gerade am Beispiel der deutschen Arbeiterschaft, die des (wenn nur partiellen) Konsenses mit dem NS-Regime zunächst ganz unverdächtig gewesen war. So wurde LUSIR - eher unbeabsichtigt - zu einer Art Gegenstudie zum zeitgleich durchgeführten Bayern-Projekt am Institut für Zeitgeschichte in München. ${ }^{44}$ Die Ergebnisse

\footnotetext{
42 Drittes Interview Ulrich Herbert mit Hermann Bergmann v. 24.2.1982, 4/15:38-16:42.

43 Vgl. Fulbrook (2011).

44 Vgl. Jureit (2007), die den Kontrast zum Bayern-Projekt hervorhebt (S. 175). Lutz Niethammer (1986a), S. 23 f., betonte diesen Unterschied schon zeitgenössisch. S. auch Oral History in der Zeitgeschichte (2017), 115-117.
} 
dieser beiden regionalgeschichtlich ausgerichteten Forschungsprojekte lagen sozusagen doppelt überkreuz: Während die Wissenschaftler in der bayerischen Hauptregion der NS-Bewegung aus den Archiven zahlreiche Evidenzen von Alltagsresistenz zutage förderten, ${ }^{45}$ schien die große Masse der Arbeiter im Revier, die nicht zur aktivistischen Speerspitze des Widerstands gehörte, ihren Frieden mit Hitler gemacht zu haben. Bei aller inneren Distanz zum Regime hatten sie von dessen sozialpolitischen, manchmal ganz handfest materiellen Segnungen profitiert; im Gegenzug verhielten sie sich still. Der grelle Unterschied in den Befunden war Resultat der heuristischen Entscheidung für unterschiedliche Quellengattungen: Am IfZ wurden Herrschaftsquellen, vor allem Polizeiberichte, ausgewertet, die gattungstypische Akte von Resistenz und Opposition dokumentierten, während die LUSIR-Mitarbeiter die Erinnerungen ehemaliger "Volksgenossen“ erfragten. Dass die Auswertung dieser lange nach den Ereignissen entstandenen Quellen dennoch ermöglichte, einen weitgehenden und Anfang der 1980er Jahre längst nicht mehr opportunen Konsens der Arbeiter mit dem NS-Regime zu belegen, spricht für die Aussagekraft dieser Quellengattung; gerade die unwillkürlichen Alltagserzählungen waren verräterisch.

Zugleich aber erschwerte die produktive Widersprüchlichkeit dieser gewissermaßen $z u$ lebendigen Quellen die historische Synthese. Anders als Schriftquellen, die sich dem interpretierenden Zugriff leichter beugen oder zu beugen scheinen (was prima vista plausibel erscheint, mag sich auf den zweiten, dritten oder vierten Blick ganz anders lesen), waren die mündlichen Erinnerungen nicht nur widersprüchlich; sie widersprachen. Die Interviewten schoben sich, wie Lutz Niethammer in seinem Aufsatz „Fragen - Antworten Fragen" so trefflich formulierte, mit "mitbestimmender Kraft" in die Perspektiven des Wissenschaftlers und entgrenzten "seine ursprüngliche Zielrichtung“: „Die Antworten stellen Fragen. ${ }^{46}$ Genau das war der Zusammenhang, auf den der auf den ersten Blick so sachlich daherkommende Aufsatztitel anspielte: nicht (nur) auf das Pingpong von Frage und Antwort im Interview, sondern auf die Entgrenzungen und Verschiebungen des Erkenntnisinteresses, die die Erinnerungstätigkeit der Gesprächspartner evozierte, und auf den programmatischen Anspruch einer solidarischen Geschichtsschreibung, mit dem LUSIR gestartet war. Gerade aber diese Volatilität der Erinnerungsforschung, die Schwierigkeit, ein Erkenntnisinteresse zu fixieren und ein konzises Argument zu formulieren, wurde als Problem empfunden. Denn die multiperspektivischen und widersprüchlichen Geschichten fügten sich kaum zu einer

45 Vgl. Wildt (2007).

$46 \quad$ Niethammer (1985b), S. 396. 
Geschichte. Wie ließ sich ihre Komplexität reduzieren, ihr Gehalt darstellerisch vermitteln? Was die historiografische Praxis bis dahin ausgemacht hatte (und bis heute ausmacht), nämlich: mit einem bestimmten Erkenntnisinteresse und unter einer bestimmten Perspektive historische Argumente zu entwickeln und zur Diskussion zu stellen, schien auf der Basis der Auswertung lebensgeschichtlicher Interviews schwierig.

Viele Jahre später, in seiner 2002 erschienenen Anthologie autobiografischer Reflexionen, gab Lutz Niethammer deswegen einer Kritik recht, die sein erster Opponent, der Bielefelder Sozialhistoriker Hans-Ulrich Wehler, auf dem Berliner Historikertag 1984 an der Alltagsgeschichte geübt hatte: dass sie nämlich nicht synthesefähig sei. ${ }^{47}$ In Ego-Histoire konzedierte Niethammer, dass die „historischen Praktiken“, die LUSIR verfolgt habe, „Anathema historischer Synthesen" seien. ${ }^{48}$ Die Interpretation lebensgeschichtlicher Interviews eröffnete aus seiner Sicht zu viele Perspektiven, Ambivalenzen und Grautöne, um sich zu Vorstellungen eines übergeordneten Geschichtsverlaufs zu fügen jedenfalls dann, wenn man sie ernst nahm und nicht nur als Illustration nutzte, wenn man sie also nicht, wie Ulrich Herbert schon 1982 formulierte, „lediglich als Steinbruch“ sah, „aus dem man sich die einzelnen Brocken zur schmückenden Illustration des großen Entwurfs mit niedlichem Detail herausklauben konnte “ ${ }^{49}$ Schon die Periodisierung machte Probleme, weil sie, wie gezeigt, mit den politischen Großzäsuren historischer Gesamtdarstellungen - 1933, 1945 nicht in eins fiel und sich zudem je nach der Gruppe, die untersucht wurde, verschob. Die Interviewpartner unterteilten ihr Leben in gute und in schlechte Zeiten - eine zyklische statt lineare Vorstellung vom Geschichtsverlauf -, aber den Zeitpunkt, wann die guten Zeiten aufhörten und die schlechten begannen, bestimmte jeder und jede anders: Männer und Frauen, die Stadt- und die Landbevölkerung, Erwachsene und Kinder, Kommunisten im Widerstand und die Masse der Arbeiter, Homosexuelle und Zwangsarbeiter, nicht-jüdische Deutsche und deutsche Juden. Wollte man also Geschichte zusammen mit ihren Subjekten schreiben, dann stand am Ende - fast vormodern - eine Vielzahl unterschiedlicher Geschichten, ein Bilderbogen heterogener historischer Erfahrungen oder eine Chronik der Erlebnisse, aber keine Synthese.

Dieses Problem der Darstellbarkeit ihrer Ergebnisse diskutierten die Alltagshistoriker schon früh. In seiner Einleitung zur Dokumentation des

47 Vgl. Wehler (1985), S. 41: „Die Alltagsgeschichte besitzt m.E. keine Synthesefähigkeit. Sie kann daher einen Gipfel der Historiographie, die große integrierende Gesamtdarstellung, nicht erklettern."

48 Vgl. Niethammer (2002), S. 133.

49 Herbert (1982), S. 14. 
„Schülerwettbewerbs Deutsche Geschichte“ 1981 forderte Ulrich Herbert, dass Geschichte „von unten“ und Geschichte „von oben“ in stetem Bezug zueinander untersucht werden müssten. Auf der einen Seite sei der "neuerdings feststellbare Trend zur Alltagsgeschichte" aus der "schrittweisen Erkenntnis“ entstanden, dass „die großen systematischen Entwürfe nicht zur Erfassung historischer Wirklichkeit taugten, wenn sie nicht vermittelt waren mit der empirischen Untersuchung kleiner sozialer Einheiten“. Auf der anderen Seite wirke der Alltag, „wenn man ihn ernsthaft untersuchte, sperrig und enttypisierend“; er lasse sich "nicht so recht“ einbinden „in einen synthetisierenden Zugriff aufs Ganze“ ${ }^{50}$ Wie aber ließen sich oben und unten, politische Entscheidungen und alltägliche Aneignungen zueinander in Beziehung setzen? Welche Textarchitektur wurde einem solchen komplexen Wechselspiel gerecht? Und inwieweit taugten die am Ende immer starr bleibenden Gliederungsentwürfe wissenschaftlicher Arbeiten überhaupt dazu, die überaus dynamischen, ständig aus der Geschichte desertierenden individuellen Geschichten abzubilden?

Die Schlussfolgerungen für das eigene Schreiben, die die LUSIR-Mitarbeiter aus dieser Problemdiagnose zogen, waren unterschiedlich. Ulrich Herbert verlor die Dimension der Alltagsgeschichte in seinen historischen Arbeiten mehr und mehr aus den Augen, obwohl er 1982 noch zuversichtlich war, dass Oral History zu „hervorragenden, mit keiner anderen Quellengattung zu erreichenden Nahanalysen sozialer Gruppen und Milieus führen" könne. ${ }^{51}$ Seine 1985 erschienene Dissertation zu den „Fremdarbeitern“, die aus seinen LUSIRForschungen zu den Arbeitern bei Krupp hervorgegangen war, diesen aber eine andere Richtung gab, war vollständig aus Archivquellen gearbeitet, auch wenn Herbert in der Einleitung darauf verwies, dass die Erfahrungen der ehemaligen Zwangsarbeiter fehlten, mittels eines großangelegten Interviewprojekts möglicherweise aber erhoben werden könnten. ${ }^{52}$ Ein solches wurde erst Jahrzehnte später im Rahmen der Entschädigung ehemaliger Zwangsarbeiter unter der Leitung von Alexander von Plato durchgeführt. ${ }^{53}$ Auch wenn die Perspektive alltäglicher Erfahrungen in Herberts Dissertation noch aufblitzte, verloren die subjektiven Quellen ihren zentralen Stellenwert. Zwar tauchte die eingangs kurz erwähnte kiebige Küchenleiterin, die aus ihrer unverhofften Leitungsfunktion den Honig eines zuvor unbekannten Machtgefühls gesogen hatte und die Herbert offensichtlich besonders negativ beeindruckt hatte, gelegentlich wieder auf, aber doch nur am Rande. Im Band Die Menschen machen

\footnotetext{
50 Herbert (1982), S. 14.

51 Ebd., S. 23.

$5^{2}$ Herbert (1985), S. 22.

53 Vgl. von Plato u.a. (2008).
} 
ihre Geschichte nicht aus freien Stücken, aber sie machen sie selbst war sie etwa auf einem unkommentiert in den Text gestellten Foto zu sehen. ${ }^{54}$ Auch in Herberts viel später geschriebenen Synthesen zur Ausländerpolitik in Deutschland oder zur deutschen Geschichte im 20. Jahrhundert spielen lebensgeschichtliche Erfahrungen nur selten, wenn überhaupt, eine Rolle, auch wenn sie möglicherweise seine Erkenntnisinteressen beeinflussten. ${ }^{55}$

Für Lutz Niethammer hingen die produktive Lernerfahrung, die LUSIR gegen den historiografischen Mainstream der siebziger und achtziger Jahre bereithielt, und das daraus resultierende Syntheseinteresse vor allem mit der konsequenten Infragestellung der Zäsur 1945 zusammen. Gegen die damals geläufige, unter anderen von Hans-Ulrich Wehler hochgehaltene Sonderwegthese, die die nationalsozialistische Machtübernahme 1933 aus tiefenhistorisch-strukturellen, sozialgeschichtlichen Ursachen erklärte und dagegen den demokratischen Neuanfang 1945 setzte, betonte er die „Kontinuität des Volkes“, die die bundesdeutsche Geschichte und Gesellschaft lange über das Kriegsende hinaus noch bestimmt habe - als es keine deutsche Nation mehr gab, deren Geschichte man hätte schreiben können. „Die Geschichte der Bundesrepublik als Demokratie“, argumentierte er in der Einführung in den ersten LUSIR-Band eindringlich, „kann nicht verstanden werden, wenn sie von der Kontinuitätsproblematik der deutschen Zeitgeschichte isoliert wird. Diese von uns und nicht von Hitler ausgehende Kontinuitätsproblematik ist von der Geschichtswissenschaft bisher stiefmütterlich behandelt worden, insgesamt ist eine Erforschung der Kontinuität des Volkes als des zweifellos auffälligsten Kontinuitätsfaktors unterblieben. ${ }^{456}$ Die erkenntnisleitende Frage der Sonderweg-Historiker, wie es „zu Hitler“ habe kommen können, sei zwar bedeutsam, verdecke jedoch „unmittelbarere, von heute aus gestellte Nachfragen an die Geschichte, nämlich wie es zu den heutigen Problemen gekommen sei“. Die „Faschismuserfahrungen“ sollten deswegen konsequent als „Vorgeschichte der Nachkriegszeit“ interpretiert werden; die Zeit nach 1945 mit all ihren deutsch-deutschen Problemlagen war der historische Fluchtpunkt. Passend dazu fand LUSIR wenige Jahre später mit der Volkseigenen Erfahrung eine DDR-Fortsetzung, und in den 2oooer Jahren plante Niethammer auf der Grundlage dieser deutsch-deutschen Forschungsprojekte eine Gesamtdarstellung der deutschen Zeitgeschichte nach 1945 im internationalen Kontext. Die Schwierigkeiten beim Schreiben einer deutschen Nationalgeschichte nach dem Zweiten Weltkrieg, die Niethammer diagnostizierte und die nicht nur, aber

\footnotetext{
54 Vgl. Herbert (1988), S. 181.

55 Vgl. Herbert (2003, 2014).

$5^{6}$ Dieses und das nächste Zitat Niethammer (1986a), S. 7 f.
} 
auch um das Problem der Synthesefähigkeit von Erfahrungsgeschichte kreisten, ließen dieses Schreibprojekt ins Stocken geraten, und schließlich brach er es ab. ${ }^{57}$ Ein breites Panorama der deutsch-deutschen Geschichte nach 1945 hat er trotzdem vorgelegt und schreibt an ihm weiter. Es setzt sich, wie ein Mosaik, aus vielen verschiedenen Texten zusammen; eine Gesamtdarstellung nicht in einem Stück, sondern als Collage.

\section{Schluss}

Aber stimmt noch heute, was sich in den achtziger Jahren andeutete, nämlich dass aus einer „von unten“ perspektivierten Erfahrungsgeschichte keine Gesamtdarstellung synthetisiert werden kann? Man könnte argumentieren, dass die heutige, kulturalistisch versierte Geschichtswissenschaft sich längst an die produktiven „Enttypisierungsschocks“ der Alltags- und Erfahrungsgeschichte, die die große Geschichte in widersprüchliche Geschichten zersprengen, gewöhnt hat. Dreißig Jahre später, heute, gehören Multiperspektivität und die widersprüchliche Synchronizität der Ereignisse und Erfahrungen, die „Gleichzeitigkeit des Ungleichzeitigen“, längst zum Mainstream und sind zu Stilmitteln historischer Darstellungen geworden. Für die breite Leserschaft historischer Sachbücher sind Zeitzeugenperspektiven (wenngleich meist in der eingangs erwähnten zerstückelten Form des histotainments) längst omnipräsent. Der O-Ton subjektiver Erfahrungen - ob aus Tagebüchern, Briefen oder eben Interviews gewonnen - wird in vielen Geschichtssynthesen (mal mehr instrumentell, mal ernsthafter und reflektierter) eingesetzt, wohl auch um die Leser bei der Stange zu halten oder die längst (zu) weit entfernte Zeit des Nationalsozialismus über die Identifikation mit subjektiven Perspektiven wieder auf Sichtweite zu holen. Diese Verlebendigung von Geschichte gelingt auch deswegen, weil der wissenschaftliche Sprachstil sich ändert, wenn man Geschichte unter dem Mikroskop von Alltagserzählungen untersucht. Er wird bildreich und farbig und auf eine oft fast literarische Weise plastisch; das zeigen die Aufsätze in den drei LUSIR-Bänden bis heute eindrücklich.

Aber auch Gesamtdarstellungen, die auf der Auswertung subjektiver Quellen basieren und aus einer Perspektive „von unten“ geschrieben sind, gibt es längst. Die zunehmende Erfahrung mit Selbstzeugnissen, darunter auch mit mündlichen oder schriftlichen Erinnerungen, hat es möglich gemacht, die Stimme des einfachen Soldaten oder der Frau von nebenan nicht nur zu illustrativen Zwecken oder zur Auflockerung in historische Narrative einzuspielen, sondern Geschichte systematisch aus dieser Perspektive zu schreiben. Häufiger 
als auf Erinnerungen greifen die Autoren dabei jedoch auf zeitgenössische Selbstzeugnisse wie Tagebücher oder Korrespondenzen zurück: Das gilt für Nicholas Stargardts 2015 erschienene Geschichte des Zweiten Weltkriegs ebenso wie für die bisher erschienenen Bände der von Norbert Frei herausgegebenen Reihe Die Deutschen und der Nationalsozialismus. ${ }^{58}$ Erinnerungen dagegen, besonders mündliche, verweisen immer auch auf andere Zeitschichten und vor allem auf die Erzählgegenwart. Sie erfordern eine besonders intensive, methodisch betriebene Quellenkritik und schicken den Interpreten zwischen der Erzählgegenwart der Interviewten und ihrer erinnerten Vergangenheit hin und her. Zwar hält dieser Prozess in der Regel herausfordernde Erkenntnisse bereit, zu einer chronologisch erzählten Geschichte indes sind mündliche Erinnerungen nur schwer zu verarbeiten. Gelingen kann eine Gesamtdarstellung aus Interviews besonders dann, wenn sich das Erkenntnisinteresse gerade auf den Prozess der Verarbeitung von (totalitären) Erfahrungen richtet, wie beispielsweise in Orlando Figes' Buch Die Flüsterer über das Privat- und Alltagsleben im Stalinismus, dessen Quellenfundament vorrangig aus Interviews besteht und das sich in einem langen letzten Kapitel den Erinnerungen selbst widmet. ${ }^{59}$

Dennoch: Dass Erfahrungen und Erinnerungen nicht nur zentraler historischer Protagonisten, sondern auch der „einfachen Leute“ Einzug in Geschichtssynthesen gehalten haben, ja dass Geschichte aus subjektiver Perspektive gedacht und geschrieben werden kann und wird, darf durchaus als ein Beleg für die langfristige Wirkung von Alltagsgeschichte und Oral History seit den achtziger Jahren verstanden werden. Mit Blick auf die NS-Geschichte sind seitdem, unter dem Label der „Volksgemeinschaft“, zunehmend Fragen nach der breiten Unterstützung des Regimes, nach Handlungsräumen und nach der agency einfacher „Volksgenossen“ ins Zentrum der Aufmerksamkeit gerückt. Wie sehr die Geschichte den Einzelnen braucht - darüber entscheiden die Fragen, die wir als Historiker und Historikerinnen oder als Geschichtsinterpreten an die Vergangenheit stellen.

\section{Literatur}

Apel, Linde (2015): „Oral History Reloaded“, in: Westfälische Forschungen 65 (2015), S. 243-254.

Büttner, Maren (2009): „Wer das Gestern versteht - kann das Morgen verändern! Deutsche Geschichtswerkstätten gestern und heute“, in: Sabine Horn/Michael

$5^{8}$ Vgl. Stargardt (2015); Roth (2015); Schanetzky (2015); Föllmer (2016); Süß (2017).

59 Vgl. Figes (2008), S. 837-916 (Kap. 9). 
Sauer (Hg.): Geschichte und Öffentlichkeit. Orte - Medien - Institutionen, Göttingen: Vandenhoeck \& Ruprecht, S. 112-120.

Einfeldt, Anne-Kathrin (1986/zuerst 1983): Auskommen - Durchkommen - Weiterkommen. Weiblicher Arbeitserfahrungen in der Bergarbeiterkolonie, in: Lutz Niethammer (Hg): „Die Jahre weiß man nicht, wo man die heute hinsetzen soll". Faschismuserfahrungen im Ruhrgebiet (LUSIR, Bd. 1), 2. Auflage, Berlin/Bonn: Dietz, S. 267-296.

Figes, Orlando (2008): Die Flüsterer. Leben in Stalins Russland. Berlin: Berlin-Verlag. Föllmer, Moritz (2016): „Ein Leben wie im Traum.“ Kultur im Dritten Reich. München: Beck.

Fulbrook, Mary (2011): Dissonant Lives. Generations and Violence Through the German Dictatorships. Oxford: Oxford University Press.

Hachtmann, Rüdiger/Reichardt, Sven: Detlev Peukert revisited. Überlegungen zu seiner historiographischen Einordnung, in dies.: (Hg.): Detlev Peukert und die NSForschung, Göttingen: Wallstein, S. 9-38.

Herbert, Ulrich (1982): „Vor der eigenen Tür. Bemerkungen zur Erforschung der Alltagsgeschichte des Nationalsozialismus“, in: Dieter Galinski/Ulrich Herbert/Ulla Lachauer (Hg.): Nazis und Nachbarn. Schüler erforschen den Alltag im Nationalsozialismus. Reinbek bei Hamburg: Rowohlt, S. 9-33.

Herbert, Ulrich (1985): „Zur Entwicklung der Ruhrarbeiterschaft 1930 bis 1960 aus erfahrungsgeschichtlicher Perspektive“, in: Lutz Niethammer/Alexander von Plato (Hg.): „Wir kriegen jetzt andere Zeiten.“ Auf der Suche nach der Erfahrung des Volkes in nachfaschistischen Ländern (LUSIR, Bd. 3). Berlin/Bonn: Dietz, S. 19-52.

Herbert, Ulrich (1985): Fremdarbeiter. Politik und Praxis des „Ausländer-Einsatzes“ in der Kriegswirtschaft des Dritten Reiches. Berlin/Bonn: Dietz.

Herbert, Ulrich (1986): „Apartheid nebenan. Erinnerungen an die Fremdarbeiter im Ruhrgebiet“, in: Lutz Niethammer (Hg.): „Die Jahre weiß man nicht, wo man die heute hinsetzen soll." Faschismuserfahrungen im Ruhrgebiet. 2. Auflage. Berlin/Bonn: Dietz, S. 233-266.

Herbert, Ulrich (1986a): „Die guten und die schlechten Zeiten“, in: Lutz Niethammer (Hg.): „Die Jahre weiß man nicht, wo man die heute hinsetzen soll.“ Faschismuserfahrungen im Ruhrgebiet. 2. Auflage. Berlin/Bonn: Dietz, S. 67-96.

Herbert, Ulrich (1988): „Europa arbeitet in Deutschland‘. Ausländische Arbeiter in der deutschen Rüstungsindustrie während des Zweiten Weltkrieges“, in: Lutz Niethammer/Bodo Hombach/Tilman Fichter u.a. (Hg.): „Die Menschen machen ihre Geschichte nicht aus freien Stücken, aber sie machen sie selbst“. Einladung zu einer Geschichte des Volkes in NRW. Berlin/Bonn: Dietz, S. 180-185.

Herbert, Ulrich (2003): Geschichte der Ausländerpolitik in Deutschland. Saisonarbeiter, Zwangsarbeiter, Gastarbeiter, Flüchtlinge. Bonn: Bundeszentrale für politische Bildung.

Herbert, Ulrich (2014): Geschichte Deutschlands im 20. Jahrhundert. München: Beck. 
Herbert, Ulrich (2015): „Arbeiterklasse und Gemeinschaftsfremde. Die Gesellschaft des NS-Staates in den Arbeiten Detlev Peukerts“, in: Rüdiger Hachtmann/Sven Reichardt (Hg.): Detlev Peukert und die NS-Forschung. Göttingen: Wallstein, S. $39-48$.

Hochmuth, Hanno (2015): „Fundstück. Theorie und Alltag. Detlev Peukert und die Geschichtswerkstätten“, in: Rüdiger Hachtmann/Sven Reichardt (Hg.): Detlev Peukert und die NS-Forschung, Göttingen: Wallstein, S. 159-174.

Jureit, Ulrike (2007): „Die Entdeckung der Zeitzeugen. Faschismus- und Nachkriegserfahrungen im Ruhrgebiet“, in: Jürgen Danyel/Jan-Holger Kirsch/Martin Sabrow (Hg.): 50 Klassiker der Zeitgeschichte. Göttingen: Vandenhoeck \& Ruprecht, S. $174-177$.

Leh, Almut (2015): „Vierzig Jahre Oral History in Deutschland. Beitrag zu einer Gegenwartsdiagnose von Zeitzeugenarchiven am Beispiel des Archivs ,Deutsches Gedächtnis“', in: Westfälische Forschungen 65 (2015), S. 255-268.

Lethen, Helmut (2012): Suche nach dem Handorakel. Göttingen: Wallstein.

Leo, Annette/Maubach, Franka (2013): Den Unterdrückten eine Stimme geben? Die International Oral History Association zwischen politischer Bewegung und wissenschaftlichem Netzwerk. Göttingen: Wallstein.

Maubach, Franka (2013): „Freie Erinnerung und mitlaufende Quellenkritik. Zur Ambivalenz der Interviewmethoden in der westdeutschen Oral History um 1980", in: BIOS 26 (2013), S. 28-52.

Maubach, Franka (2017): „Unerhörte Begebenheiten. LUSIR und die Innovationskraft der frühen Oral History“, in: Zeitgeschichte in Hamburg, S. 12-26.

Medjedović, Irena (2014): Qualitative Sekundäranalyse. Zum Potenzial einer neuen Forschungsstrategie in der empirischen Sozialforschung. Wiesbaden: Springer VS.

Medjedović, Irena/Witzel, Andreas (2010): Wiederverwendung qualitativer Daten: Archivierung und Sekundärnutzung qualitativer Interviewtranskripte. Wiesbaden: Springer VS.

Morat, Daniel (2015): „Geschichte hören. Zum quellenkritischen Umgang mit historischen Tondokumenten“, in: Geschichte in Wissenschaft und Unterricht 66 (2015), S. $703-726$.

Niethammer, Lutz (Hg.) (1983): „Hinterher merkt man, daß es richtig war, daß es schiefgegangen ist.' Nachkriegs-Erfahrungen im Ruhrgebiet". Berlin/Bonn: Dietz.

Niethammer, Lutz/von Plato, Alexander (Hg.) (1985): „Wir kriegen jetzt andere Zeiten.“ Auf der Suche nach der Erfahrung des Volkes in nachfaschistischen Ländern (LUSIR, Bd. 3). Berlin/Bonn: Dietz.

Niethammer, Lutz/von Plato, Alexander: „Vorwort“, in dies. (Hg.) (1985a): „Wir kriegen jetzt andere Zeiten." Auf der Suche nach der Erfahrung des Volkes in nachfaschistischen Ländern (LUSIR, Bd. 3). Berlin/Bonn: Dietz, S. 9-15.

Niethammer, Lutz (1985b): „Fragen - Antworten - Fragen. Methodische Erfahrungen und Erwägungen zur Oral History“, in: ders./von Plato, Alexander (Hg.):„Wir kriegen 
jetzt andere Zeiten." Auf der Suche nach der Erfahrung des Volkes in nachfaschistischen Ländern (LUSIR, Bd. 3), Berlin/Bonn: Dietz, S. 392-445.

Niethammer, Lutz (Hg.) (1986, zuerst 1983): „Die Jahre weiß man nicht, wo man die heute hinsetzen soll“. Faschismuserfahrungen im Ruhrgebiet (LUSIR, Bd. 1). 2. Auflage. Berlin/Bonn: Dietz.

Niethammer, Lutz (1986a): „Einleitung des Herausgebers“, in ders. (Hg.): „Die Jahre weiß man nicht, wo man die heute hinsetzen soll“. Faschismuserfahrungen im Ruhrgebiet. 2. Auflage. Berlin/Bonn: Dietz, S. 7-29.

Niethammer, Lutz (1986b): „Heimat und Front. Versuch, zehn Kriegserinnerungen aus der Arbeiterklasse des Ruhrgebiets zu verstehen“, in ders. (Hg.): „Die Jahre weiß man nicht, wo man die heute hinsetzen soll“. Faschismuserfahrungen im Ruhrgebiet. 2. Auflage. Berlin/Bonn: Dietz, S. 163-232.

Niethammer, Lutz/Hombach, Bodo/Fichter, Tilman, et al. (Hg.) (1988): „Die Menschen machen ihre Geschichte nicht aus freien Stücken, aber sie machen sie selbst“. Einladung zu einer Geschichte des Volkes in NRW. Berlin/Bonn: Dietz.

Niethammer, Lutz (1991): „Glasnost privat 1987“, in ders./Alexander von Plato/Dorothee Wierling: Die volkseigene Erfahrung. Eine Archäologie des Lebens in der Industrieprovinz der DDR. 3 o biographische Eröffnungen. Berlin: Rowohlt, S. 9-73.

Niethammer, Lutz (1999): „Schwierigkeiten beim Schreiben einer deutschen Nationalgeschichte nach dem Zweiten Weltkrieg“, in ders.: Deutschland danach. Postfaschistische Gesellschaft und nationales Gedächtnis. Bonn: Dietz, S. 434-449.

Niethammer, Lutz (2002): Ego-Histoire? Und andere Erinnerungsversuche. Wien u.a.: Böhlau.

Niethammer, Lutz (2013): „Nachwort“, in: Annette Leo/Franka Maubach (Hg.): Den Unterdrückten eine Stimme geben? Die International Oral History Association zwischen politischer Bewegung und wissenschaftlichem Netzwerk. Göttingen: Wallstein, S. 291-318.

Niethammer, Lutz „Oral History in der deutschen Zeitgeschichte. Interview von Veronika Settele und Paul Nolte mit Lutz Niethammer“ (2017), in: Geschichte und Gesellschaft 43 (2017), 110-145.

Paul, Gerhard/Schock, Ralph (2014) (Hg.): Sound der Zeit. Geräusche, Töne, Stimmen 1898 bis heute. Göttingen: Wallstein.

Peukert, Detlev (1976): Ruhrarbeiter gegen den Faschismus. Dokumentation über den Widerstand im Ruhrgebiet 1933-1945. Frankfurt a. M.: Röderberg.

Peukert, Detlev (1980): Die KPD im Widerstand. Verfolgung und Untergrundarbeit an Rhein und Ruhr 1933-1945. Wuppertal: Hammer.

Peukert, Detlev (1982): Volksgenossen und Gemeinschaftsfremde: Anpassung, Ausmerze und Aufbegehren unter dem Nationalsozialismus. Köln: Bund.

Plato, Alexander von (1986): „Ich bin mit allen gut ausgekommen‘. Oder: war die Ruhrarbeiterschaft vor 1933 in politische Lager zerspalten?“, in: Lutz Niethammer (Hg.): 
„Die Jahre weiß man nicht, wo man die heute hinsetzen soll“. Faschismuserfahrungen im Ruhrgebiet. 2. Auflage. Berlin/Bonn: Dietz, S. 31-65.

Plato, Alexander von/Leh, Almut/Thonfeld, Christoph (Hg.) (2008): Hitlers Sklaven. Lebensgeschichtliche Analysen zur Zwangsarbeit im internationalen Vergleich. Wien: Böhlau.

Raulff, Ulrich (2014): Wiedersehen mit den Siebzigern. Die wilden Jahre des Lesens. Stuttgart: Klett-Cotta.

Roth, Markus (2015): „Ihr wisst, wollt es aber nicht wissen." Verfolgung, Terror und Widerstand im Dritten Reich. Beck: München.

Sabrow, Martin/Frei, Norbert (Hg.) (2012): Die Geburt des Zeitzeugen nach 1945. Göttingen: Wallstein.

Schanetzky, Tim (2015): „Kanonen statt Butter“. Wirtschaft und Konsum im Dritten Reich. München: Beck.

Schöttler, Peter (1984): „Die Geschichtswerkstatt e.V. Zu einem Versuch, basisdemokratische Geschichtsinitiativen und -forschungen zu vernetzen“, in: Geschichte und Gesellschaft 10 (1984), S. 420-424.

Schütze, Fritz (1978): Die Technik des narrativen Interviews in Interaktionsfeldstudien. Arbeitsberichte und Forschungsmaterialien der Fakultät für Soziologie der Universität Bielefeld, Nr. 1. 2. Auflage. Bielefeld o. V.

Schütze, Fritz (1981): „Prozeßstrukturen des Lebenslaufs“, in: Joachim Mathes/Arno Pfeifenberger/Manfred Stoßberg (Hg.): Biographie in handlungswissenschaftlicher Perspektive. Nürnberg: Verlag der Nürnberger Forschungsvereinigung.

Stargardt, Nicholas (2015): Der deutsche Krieg 1939-1945. Frankfurt a. M.: Fischer.

Süß, Dietmar (2017): „Ein Volk, ein Reich, ein Führer“. Die deutsche Gesellschaft im Dritten Reich. Beck: München.

Wachsmann, Nicolaus: Rewriting resistance and repression under the Nazi regime. Perspectives on the work of Detlef J.K. Peukert, in: Rüdiger Hachtmann/Sven Reichardt (Hg.): Detlev Peukert und die NS-Forschung, Göttingen: Wallstein, S. 102-114.

Wehler, Hans-Ulrich (1985): „Königsweg zu neuen Ufern oder Irrgarten der Illusionen? Die westdeutsche Alltagsgeschichte: Geschichte ,von innen' und ,von unten“', in: Franz Josef Brüggemeier/Jürgen Kocka (Hg.): Geschichte von unten - Geschichte von innen. Kontroversen um die Alltagsgeschichte. Hagen: FernUniversität.

Wildt, Michael (2007): „Das ,Bayern-Projekt', die Alltagsforschung und die ,Volksgemeinschaft“", in: Norbert Frei (Hg.): Martin Broszat, der „Staat Hitlers“ und die Historisierung des Nationalsozialismus. Göttingen: Wallstein, S. 119-129.

Zimmermann, Michael (1986): „Ausbruchshoffnung. Junge Bergleute in den Dreißiger Jahren“, in: Lutz Niethammer (Hg.): „Die Jahre weiß man nicht, wo man die heute hinsetzen soll“. Faschismuserfahrungen im Ruhrgebiet. 2. Auflage. Berlin/Bonn: Dietz, S. $97-132$. 